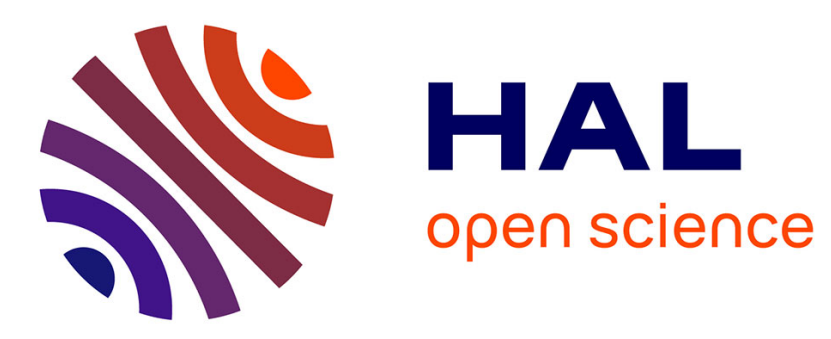

\title{
The sensitivity of a model of low stratiform clouds to radiation
}

C. Fravalo, Yves Fouquart, R. Rosset.

\section{To cite this version:}

C. Fravalo, Yves Fouquart, R. Rosset.. The sensitivity of a model of low stratiform clouds to radiation. Journal of the Atmospheric Sciences, 1981. hal-01945280

\section{HAL Id: hal-01945280 \\ https://hal.uca.fr/hal-01945280}

Submitted on 8 Jun 2021

HAL is a multi-disciplinary open access archive for the deposit and dissemination of scientific research documents, whether they are published or not. The documents may come from teaching and research institutions in France or abroad, or from public or private research centers.
L'archive ouverte pluridisciplinaire HAL, est destinée au dépôt et à la diffusion de documents scientifiques de niveau recherche, publiés ou non, émanant des établissements d'enseignement et de recherche français ou étrangers, des laboratoires publics ou privés. 


\title{
The Sensitivity of a Model of Low Stratiform Clouds to Radiation
}

\author{
C. Fravalo \\ Université de Bretagne Occidentale, Brest, France \\ Y. FouquarT \\ Université des Sciences et Techniques de Lille I, France
}

R. ROSSET

Université de Clermont II, France

(Manuscript received 19 December 1979, in final form 12 February 1981)

\begin{abstract}
The low stratiform cloud model presented here appears as a generalization of Lilly's model (1968). Its main new apsects lie 1) in a detailed vertical computation of the longwave and the shortwave radiative flux profiles and 2) in a formulation of the entrainment rate and the turbulent flux profiles which takes into account the nonlinear vertical structure of the radiative fluxes. Furthermore, the radiative divergence is no longer externally prescribed, like in other models; it is determined as function of the mixed-layer and cloud characteristics. All these features allow a more complete coupling between the turbulent mixing and the radiative fluxes. Within the cloud, the turbulent flux profiles of the moist static energy and the virtual dry static energy are nonlinear functions of height, due to the radiative divergence. This nonlinear structure results in a realistic negative entrainment flux at cloud top.

In the sensitivity tests, the stress has been put on the variability of the radiative and turbulent fluxes as functions of the cloud microphysics. The result is that the integrated liquid water content (liquid water path) is the predominant factor in fixing the radiative and turbulent fluxes, with a secondary but non-negligible role played by the drop size distribution.

For optically thin clouds where the shortwave absorption is negligible, the infrared cooling is distributed throughout the whole cloud and is highly sensitive to the liquid water path; thus the turbulent fluxes and the entrainment rate also depend strongly on the liquid water path.

When increasing the liquid water path, the longwave cooling becomes saturated and localized in a layer of progressively reduced thickness at cloud top. Thus for thick clouds, with no solar flux, our model gives results which are similar to those of Lilly-type models.

The solar heating does not saturate as the liquid water path increases. Moreover, since it is distributed throughout the cloud deck, it not only reduces the radiative divergence and the turbulent kinetic energy production at cloud top, but it also acts as a source of this latter energy component near the cloud base. The overall result is a noticeable reduction of the entrainment rate. This suggests a strong diurnal cycle for thick stratocumulus decks.
\end{abstract}

\section{1. 甚保roduction}

Clouds act as a very efficient modulator of the earth's radiation budget. Depending on their type and temperature, they can have either a positive or a negative effect on the radiative balance. Extended persistent clouds of the mixed layer are particularly important in this context and their parameterization is a critical point in climate modeling.

The state of equilibrium of a cloud-topped mixed layer is determined by competition between radiative cooling, entrainment of warm and dry air from above the cloud, large-scale divergence and turbulent buoyancy fluxes. Entrainment is itself dependent on the radiative fluxes which, in turn, depend on thermodynamical and microphysical cloud properties.
For Lilly (1968), Schubert (1976) and Kraus and Schaller (1978a) (hereafter denoted as Lilly-îye models), the radiative cooling was assumed to be confined to an infinitesimally thin inversion layer located just above the cloud top, so that it did not contribute to the generation of turbulence in the mixed layer. On the contrary, for Kahn and Businger (1979), the mixed layer is driven convectively by radiative cooling which occurs in the layer $\sim 100 \mathrm{~m}$ thick in the upper part of the cloud deck. This latter assumption is in better agreement with the observations of Paltridge (1971, 1974a), Platt (1976) and Stephens et al. (1978) who reported radiative divergence layers with thicknesses largely exceeding $100 \mathrm{~m}$. 


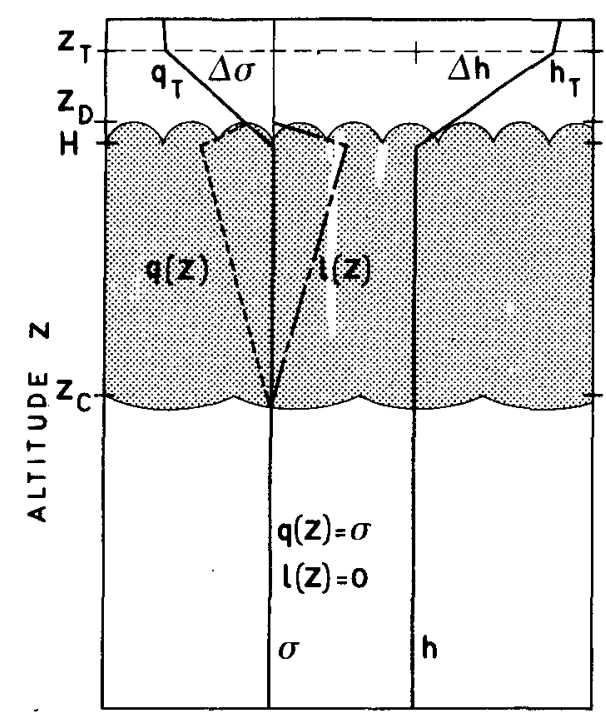

FIG. 1. Schematic profiles assumed for $h$ and $\sigma$ and cloud profiles of $q(z)$ and $l(z)$. For clarity of presentation, the slopes of the lines $q(z)$ and $l(z)$ are considerably exaggerated. The jumps $\Delta h$ and $\Delta \sigma$ are located in the inversion zone between $z_{T}$ and $H$. For radiative computations (Section 3) six layers are considered between cloud base $z_{C}$ and level $z_{D}$, the mean height of the upper excursions of the cloud top.

In order to account better for these observations, Deardorff (1976) and Schubert et al. (1979) spread radiative cooling vertically over both the inversion and the mixed layers. However, the adopted partition is still arbitrary and the effect of the radiative divergence on the entrainment and the turbulent flux profiles is only partially considered.

More recently, Randall (1980a) emphasized the interaction between radiative fluxes, entrainment and turbulent flux profiles. He showed that a cloudtopped mixed-layer model is highly sensitive to the thickness of the radiative divergence zone, particularly over cold seas and/or in the presence of strong subsidence.

The partition of the radiative divergence between the mixed and the inversion layers is obviously arbitrary. To avoid this difficulty we compute explicitly the solar and the infrared flux profiles within these two layers.

In Section 2 we present a formulation for the turbulent fluxes and the entrainment rate. The computational procedure for the radiative flux profiles is described in Section 3. In Section 4, with a diagnostic version of our model, we used Lilly's 1968 experimental data to calibrate the abovementioned procedures. We then made tests of the effect of the location and the intensity of the radiative divergence on the turbulent flux profiles and the entrainment rate (Section 5). These tests were carried out by simulating variations in the microphysical cloud properties; they clearly show the strong coupling between radiation and turbulent mixing in low stratiform clouds.

\section{Formulation of entrainment rate and turbulent flux profiles}

A schematic representation of the cloud-topped mixed layer is given in Fig. 1. Due to turbulent mixing, the moist static energy $h=C_{p} T+g z+L q$ and the sum $\sigma=q+l$ of the water vapor $q$ and liquid water $l$ mixing ratios are considered as conservative quantities within the mixed layer. In these two definitions the other quantities have their usual meanings. The top of the mixed layer, which coincides with the mean cloud top, is $H$ while $z_{C}$ is the condensation level. The inversion zone is located between level $H$ and top level $z_{T}$. Within this zone, we define discontinuities in $h$ and $\sigma$, respectively, $\Delta h=h_{T}-h$ and $\Delta \sigma=q_{T}-\sigma$, where $h_{T}$ and $q_{T}$ are externally specified parameters at level $z_{T}$. Small convective cloud turrets of mean cloud-top altitude $z_{D}$ penetrate into the inversion zone (Deardorff, 1976).

\section{a. Entrainment hypothesis}

Let $s_{v}=C_{p} T(1+\delta q-l)$ be the virtual dry static energy $(\delta=0.608)$ and $F_{s v}(z)$ be its vertical turbulent flux at level $z$. A formal integration of $F_{s v}(z)$ through the mixed layer gives the integral

$$
J=H^{-1} \int_{0}^{H} F_{s v}(z) d z=P+N,
$$

where $P$ and $N$, the positive and negative components of $J$, are proportional, respectively, to the production and destruction terms of the turbulent kinetic energy in the mixed layer.

Following Kraus and Schaller (1978b) and neglecting any turbulent mechanical term, we assume a constant ratio $k^{2}$ between $|N|$ and $P$. This entrainment hypothesis can be written as

$$
k^{2} P+N=0 .
$$

The exact value of $k$ is not well known (Deardorff, 1980b). However, for the time being, we take $k=0.2$, corresponding to a clear convective mixed layer.

\section{b. Turbulent and radiative fluxes}

The solution of (2.1) requires knowledge of the turbulent buoyancy flux profile $F_{s v}(z)$. The relations between $F_{s v}(z)$ and the turbulent fluxes of moist static energy $\left[F_{h}(z)\right]$ and total water mixing ratio $\left[F_{\sigma}(z)\right]$ were first established by Lilly (1968). Using Schubert's (1976) formalism and notations, they are

$$
F_{s v}(z)=F_{h}(z)-(1-\delta \epsilon) L F_{o}(z)
$$


in the clear atmosphere below the cloud base and

$$
F_{s v}(z)=\beta F_{h}(z)-\epsilon L F_{\sigma}(z)
$$

within the saturated cloudy atmosphere. The parameters $\epsilon$ and $\beta$ are defined in Schubert (1976). They vary slowly with height. In the following discussion we calculate them at the condensation level only.

Since $\sigma$ is constant with height, its turbulent flux must be linear with respect to $z$ so that

$$
F_{\sigma}(z)=\left(F_{\sigma}\right)_{S}-z H^{-1}\left[\left(F_{\sigma}\right)_{S}-\left(F_{\sigma}\right)_{H}\right],
$$

where, the subscripts $S$ and $H$ refer to the surface and to the top of the mixed layer, respectively.

Eqs. (2.1)-(2.4) are commonly used in all Lillytype models. Another fundamental assumption is that the radiative flux divergence occurs entirely in the inversion zone and not in the mixed layer. In this paper, in order to test the influence of the radiative process upon the turbulent fluxes, we take into account the radiative divergence which occurs in the mixed layer.

Assuming no horizontal advection, the conservation equation of $h$ is

$$
\rho \frac{\partial h}{\partial t}=-\frac{\partial}{\partial z}\left[F_{h}(z)+R(z)\right]
$$

where $\rho$ is the air density and $R(z)$ the net radiative flux at level $z$, including the longwave flux $R L(z)$ and the shortwave flux $R S(z)$ so that

$$
R(z)=R L(z)+R S(z) .
$$

Since $h$ is independent of $z$, it clearly comes out that it is the sum $F_{h}(z)+R(z)$ which is linear with respect to $z$ and not $F_{h}(z)$ alone as assumed in Lillytype models. This argument leads us to write

$$
\begin{aligned}
F_{h}(z)=\left(F_{h}\right)_{S}-z & H^{-1}\left[\left(F_{h}\right)_{S}-\left(F_{h}\right)_{H}\right. \\
& \left.+R_{S}-R_{H}\right]+R_{S}-R(z) .
\end{aligned}
$$

If the radiative flux is assumed constant in the mixed layer as in Lilly-type models $\left[R(z)=R_{S}\right.$ $=R_{H}$ ], all the radiative terms vanish in (2.5) and $F_{h}(z)$ and thus $F_{s v}(z)$ become linear.

On the contrary, in the present paper, we relax this oversimplification by calculating the net radiative fluxes at different levels, as functions of the mixed-layer and cloud characteristics. Thus in the cloud, the divergence of the radiative profile $R(z)$ leads to a nonlinear structure for $F_{h}(z)$ in (2.5) as well as for $F_{s v}(z)$ in (2.3). This allows a more complete interaction between radiative and turbulent fluxes.

To close the system $(2.1)-(2.5)$, we express the turbulent fluxes $\left(F_{h}\right)_{H}$ and $\left(F_{\sigma}\right)_{H}$ at level $H$ in terms of the entrainment rate $w_{e}$ (Lilly, 1968)

$$
\begin{aligned}
& \left(F_{h}\right)_{H}=-\rho w_{e} \Delta h+R_{T}-R_{H}, \\
& \left(F_{\sigma}\right)_{H}=-\rho w_{e} \Delta \sigma .
\end{aligned}
$$

In (2.6) $R_{T}-R_{H}$ is the net radiative heat loss of the inversion layer, mostly due to the liquid water content of the cloud turrets.

Finally, in the present model, the radiative divergence occurs in the mixed layer [see Eq. (2.5)] as well as in the inversion zone [see Eq. (2.6)] since as reported by Deardorff (1980b) ". . . it does not appear reasonable to assume that all of it occurs either in the well-mixed layer or in the capping inversion alone."

\section{c. Upper and lower limits for $w_{e}$}

The vertical integration of the buoyancy flux within the mixed layer is found from Eqs. (2.2)(2.7) and we may write the integral $J$ as

$J=1 / 2\left(A_{1}\left(F_{\text {sv }}^{*}\right)_{S}+A_{2}\left(F_{\text {sv }}\right)_{S}+J_{R}\right.$

$$
\begin{aligned}
& -\rho w_{e}\left\{\left(\beta A_{4}+A_{3}\right) \Delta h\right. \\
& \left.\left.-\left[\epsilon A_{4}+(1-\delta \epsilon) A_{3}\right] L \Delta \sigma\right\}\right),
\end{aligned}
$$

with

$$
\begin{aligned}
& A_{1}=\left(1-\frac{z_{C}}{H}\right)^{2}=1-A_{2} \\
& A_{3}=\left(\frac{z_{C}}{H}\right)^{2}=1-A_{4}
\end{aligned}
$$

and

$$
\left.\begin{array}{l}
\left(F_{s v}^{*}\right)_{S}=\beta\left(F_{h}\right)_{S}-\epsilon L\left(F_{\sigma}\right)_{S} \\
\left(F_{s v}\right)_{S}=\left(F_{h}\right)_{S}-(1-\delta \epsilon) L\left(F_{\sigma}\right)_{S}
\end{array}\right\} .
$$

In $(2.10),\left(F_{s v}^{*}\right)_{S}$ and $\left(F_{s v}\right)_{S}$ represent the surface fluxes of $s_{v}$ in cloudy (2.3) and clear (2.2) conditions, respectively.

In (2.8), $J_{R}$ is the contribution of the radiative terms to the net turbulence with

$$
\begin{aligned}
J_{R}=\left(\beta A_{4}+A_{3}\right) & \left(R_{T}-R_{S}\right)-2 H^{-1} \int_{0}^{z_{C}}\left[R(z)-R_{S}\right] d z \\
& -2 \beta H^{-1} \int_{z_{C}}^{H}\left[R(z)-R_{S}\right] d z .
\end{aligned}
$$

Our computations show that the contribution of the radiative fluxes to the turbulence is a function of both the overall difference $R_{T}-R_{S}$ and the integrated value of $R(z)-R_{S}$, while in Lilly-type models this last contribution is always neglected.

In the present study the net turbulence within the mixed layer is non-negative $(J \geqslant 0)$. The upper limit of the entrainment rate is thus given by the condition $J=0$ (Ball, 1960), viz.,

$$
\left(w_{e}\right)_{\max }=\frac{A_{1}\left(F_{s v}^{*}\right)_{S}+A_{2}\left(F_{s v}\right)_{S}+J_{R}}{\rho\left\{\left(\beta A_{4}+A_{3}\right) \Delta h-\left[\epsilon A_{4}+(1-\delta \epsilon) A_{3}\right] L \Delta \sigma\right\}} .
$$


Practically, the condition $\left(w_{e}\right)_{\max }>0$ is always satisfied for stable stratocumulus situation.

Moreover, to prevent instabilities at the inversion level, $\left(F_{s v}\right)_{H}$ must remain non-positive, whereas the quantity $\beta \Delta h-\epsilon L \Delta \sigma$ must be positive (Randall, 1980b; Deardorff, 1980a). Using (2.6), (2.7) and (2.3), we have

$$
\left(F_{s v}\right)_{H}=\beta\left(R_{T}-R_{H}\right)-\rho w_{e}(\beta \Delta h-\epsilon L \Delta \sigma) .
$$

Since $R_{T}-R_{H}$ is always positive, the minimum entrainment rate is defined by

$$
\left(w_{e}\right)_{\min }=\frac{\beta\left(R_{T}-R_{H}\right)}{\rho(\beta \Delta h-\epsilon L \Delta \sigma)} .
$$

\section{d. Algorithm for calculating the turbulent fux profiles and the entrainment velocity}

With the entrainment hypothesis formulated in (2.1), and assuming that the surface fluxes $\left[\left(F_{h}\right)_{S}\right.$ and $\left(F_{\sigma}\right)_{S}$, the mixed-layer and cloud characteristics and the net radiative flux profiles are known, the iterative procedure we propose for calculating the turbulent flux profiles and $w_{e}$ is as follows:

(i) Perform numerical integration of the difference $R(z)=\left[R S(z)-R S_{S}\right]+\left[R L(z)-R L_{S}\right]$ and $\cdot$ calculate $J_{R}$ from (2.11).

(ii) Apply (2.12) to derive $\left(w_{e}\right)_{\max }$ and (2.14) to derive $\left(w_{e}\right)_{\min }$. Note that for $w_{e}=\left(w_{e}\right)_{\max }, P=-N$ $(J=0)$, leading to $k^{2} P+N<0$ since $k^{2} \ll 1$.

(iii) Initialize $w_{e}=\left[\left(w_{e}\right)_{\max }+\left(w_{e}\right)_{\min }\right] / 2$.

(iv) Derive $\left(F_{h}\right)_{H}$ and $\left(F_{\sigma}\right)_{H}$ from (2.6) and (2.7), $F_{\sigma}(z)$ and $F_{h}(z)$ from (2.4) and (2.5) and finally, the profile $F_{s v}(z)$ from (2.2) and (2.3).

(v) Determine the region(s) of negative $F_{s v}(z)$.

(vi) Calculate $J$ from (2.8) and perform the numerical integration of the negative part of $F_{s v}(z)$ to obtain $N$ and then $P=J-N$.

(vii) Find the sign of $k^{2} P+N$ and then find $w_{e}$ by a dichotomic process repeating steps (iv)-(vii) until (2.1) is satisfied.

If the condition $\left(w_{e}\right)_{\max } \geqslant\left(w_{e}\right)_{\min }$ is satisfied there exist a unique solution for $w_{e}$ which satisfies (2.1) together with $J \geqslant 0$ and $\left(F_{s v}\right)_{H} \leqslant 0$.

Our algorithm is no longer relevant in two cases:

1) When the inversion strength decreases $(\Delta h$ and/or $\Delta \sigma \rightarrow 0$ ). In this case, the denominator in (2.14) decreases faster than in (2.12): this results in $\left(w_{e}\right)_{\min }>\left(w_{e}\right)_{\max }$. This situation corresponds to an intense entrainment leading to rapid dissipation of the cloud cover.

2) When the production of turbulent kinetic energy as expressed by the first three terms in (2.8) weakens (e.g., over very cold seas and/or for very weak radiative cooling). This occurs for large $H$ values and thin clouds. In this case, the energy budget should include the mechanical terms.

\section{Parameterization of radiative fluxes}

To test the sensitivity of the model to both the location and intensity of the radiative divergence, we must determine the radiative flux profiles $R S(z)$ (shortwave) and $R L(z)$ (longwave) and their dependence on moist air characteristics.

\section{a. Cloud characteristics}

There is experimental evidence of vertical gradients in both liquid water contents $\rho l(z)$ and drop size distributions in low stratiform clouds (Paltridge, 1974a; Stephens et al., 1978). To account for these variations, we divide our modeled cloud into six layers. We must then specify in each cloud layer, temperature $T$, water vapor $q$ and liquid water $l$ mixing ratios and the drop size distribution.

In the cloud, the vertical temperature gradient is moist adiabatic. As a consequence of the basic hypothesis of the model (see Section 2 and Fig. 1), the profiles of the water vapor and the liquid water mixing ratios are fixed: these two quantities increase linearly with height as shown in Fig. 1. However, observed liquid water contents (Paltridge, 1974a) are much lower than their predicted adiabatic values.

Part of this discrepancy is probably due to a systematic underestimate of the liquid water content measurements which are notoriously difficult. However, the entrainment of dry air from above the cloud may considerably reduce the liquid water content principally near the cloud top. For simplicity we assume that this reduction affects the cloud everywhere and we introduce a constant "diabatism coefficient" $A$, equal to the ratio between real and adiabatic $(A=1)$ liquid water mixing ratios. Obviously, this coefficient must be adjusted using statistics based on the mean cloud deck altitude, cloud age and entrainment intensity. In the following, different values of $A$ are specified in our sensitivity tests.

Using the Clausius-Clapeyron equation, the liquid water path for each cloud layer of thickness $\Delta z$ is

$$
W=\int_{\Delta z} \rho l(z) d z=\frac{g \rho \alpha}{2 L(1+\gamma)} A(\Delta z)^{2},
$$

where $\gamma=L^{\dot{2}} q^{*} /\left(R_{v} C_{p} T^{2}\right)$ and $\alpha=\gamma-\dot{L} q^{*} /\left(R_{d} T\right)$, with $q^{*}$, the saturated value of $\sigma$ at temperature $T$, and $R_{d}$ and $R_{v}$ the gas constants for dry air and water vapor, respectively.

The cloud radiative properties are defined by the extinction and the scattering cross sections and the scattering function. According to Mie theory, these parameters are complex functions of wavelength and drop spectrum. In order to save computational time, the drop spectrum of each cloud layer is repre- 
sented by its effective mean radius

$$
r_{e}=\int r^{3} n(r) d r\left[\int r^{2} n(r) d r\right]^{-1},
$$

where $n(r)$ is the spectral density at radius $r$. This is a crude but reasonable approximation since the cloud optical thickness $\tau$ in the shortwave region is related to $r_{e}$ by $\tau=3 W / 2 r_{e}$ (Stephens, 1978b). This result is also consistent with the use of approximate methods of resolution of the radiative transfer equation. The scattering parameters are calculated from Mie theory for monodispersed distributions of radius $r_{e}$.

Most of the published experimental drop spectra (Feigelson, 1964; Paltridge, 1974a) show that both the liquid water mixing ratio $l$ and the mode radius $r_{m}$ of the drop spectrum increase with height. After Paltridge (1974a), we adopt the empirical relationship (with $r_{m}$ in $\mu \mathrm{m}$ )

$$
r_{m}=30 \rho l+b .
$$

The value $b=2 \mu \mathrm{m}$ is typical of marine stratocumulus (Stephens, 1978a). Furthermore, assuming a modified gamma distribution, $r_{e}$ and $r_{m}$ are related by $r_{e}=3 / 2 r_{m}$, thus giving a linear increase for $r_{e}$ from the base to the cloud top.

The microphysical and thermodynamical cloud properties are now completely defined. The impact of variations of the microphysical properties on the cloud model can then be evaluated in terms of fluctuations of the two empirical parameters $A$ and $b$ which determine the liquid water path $(A)$ and the drop spectrum $(A$ and $b$ ). For further convenience in the discussions of Sections 4 and 5, we adopt a unique equivalent mean radius $\bar{r}_{e}$, averaged over the whole cloud and defined as $\bar{r}_{e}=3 W / 2 \tau_{N}$, where $W$ has been calculated in (3.1) with $\Delta z=z_{D}-z_{C}$ and where $\tau_{N}$ is the total cloud optical thickness in the visible range $(\lambda=0.5 \mu \mathrm{m})$.

Finally, the moist static energy $h$ and the water vapor mixing ratio $q$ are fixed at their (unique) mixed-layer values below the cloud. Above the $z_{T}$ level, $h(z)$ and $q(z)$ are assumed linear, rejoining the standard atmosphere at $5000 \mathrm{~m}$.

\section{b. Radiative transfer}

The radiative transfer equation defining the diffuse radiance $I$ at frequency $\nu$ is

$$
\begin{aligned}
\mu & \frac{d I(\nu, \mu)}{d \tau} \\
=-I_{\nu}(\tau, \mu) & +1 / 2 \omega_{\nu} \int_{-1}^{+1} \Gamma_{\nu}\left(\mu, \mu^{\prime}\right) I_{\nu}\left(\tau, \mu^{\prime}\right) d \mu^{\prime} \\
& +1 / 2 \omega_{\nu} F_{\nu} \Gamma_{\nu}\left(\mu, \mu_{0}\right) \exp \left(-\tau_{\nu} / \mu_{0}\right) .
\end{aligned}
$$

In this expression $\mu=\cos \theta$, where $\theta$ is the zenith angle and $\pi F_{v}$ the incident solar irradiance in the direction $\mu_{0}=\cos \theta_{0}$. We also define $K_{\nu}(z)$ the extinction coefficient,

$$
\tau=\int_{z_{D}}^{z} K_{\nu}(z) d z
$$

the optical depth, obviously frequency dependent, $\omega_{\nu}=\xi_{\nu} / K_{\nu}$ the single-scattering albedo, with $\xi_{\nu}$ the scattering coefficient and $\Gamma_{v}\left(\mu, \mu^{\prime}\right)$ the scattering phase function defining the incident light at $\mu^{\prime}$ which is scattered in the direction $\mu$. For longwave radiation, where thermal emission is important and solar radiation negligible, a term $\left(1-\omega_{\nu}\right) B_{\nu}(\tau)$ with $B_{\nu}(\tau)$ the Planck function at temperature $T(\tau)$ replaces the last quantity in (3.2).

The net radiative flux is

$$
R(\tau)=\int_{0}^{\infty} R_{\nu}(\tau) d \nu=2 \pi \int_{0}^{\infty} \int_{-1}^{+1} \mu I(\nu, \mu) d \mu d \nu
$$

Approximate methods of resolution of (3.2) are generally of poor accuracy. with regard to the radiance field, but in the present case we are concerned with the radiative flux, for which these methods generally yield results which agree within $5 \%$ with exact results (Lenoble, 1977). More precisely, the disagreement between exact and approximate results is particularly noticeable in the solar range for strong absorption. This is a consequence of the marked anisotropy of the scattering phase function. In the thermal infrared, the accuracy is much better, mostly because the source function $\left(1-\omega_{v}\right) B_{\nu}(\tau)$ is isotropic, but also because the scattering phase functions are much smoother at these wavelengths. Basically, we retain the same method for both solar and thermal infrared and use the Eddington approximation in a version similar to that of Shettle and Weinmann (1970). However, we take into account for the anisotropy of the phase function in the solar range for which we use a method called "double- $\delta$ Eddington" by Lenoble (1977).

\section{c. Shortwave radiation}

Only scattering and absorption by atmospheric gases and particles occur in the shortwave region. Nevertheless, the strong anisotropy of the scattering function and the interactions between scattering and molecular absorption need special attention.

In the present model, the shortwave radiative fluxes are calculated using a method described by Fouquart and Bonnel (1980). The major difference with Fouquart and Bonnel's parameterization is that for uniformity with the longwave range, we use the double- $\delta$ Eddington approximation instead of the exponential kernel. Tests of the accuracy of the double- $\delta$ Eddington approximation can be found in Lenoble (1977) and this method appears to be one of 
the best approximate methods. Since this method has never been presented, we find it necessary to discuss it here.

As shown by Wiscombe and Joseph (1977), the Eddington approximation predicts cloud albedo and absorptivity reasonably well for phase functions having the asymmetry factor $\langle\cos \theta\rangle \leqslant 0.5$. Now, the intensity of the diffraction peak increases with the Mie parameter $x=2 \pi r / \lambda$. Thus, in the shortwave region, cloud scattering phase functions exhibit a very sharp peak for small scattering angles, giving high values of the asymmetry factor. Improved approximated methods such as $\delta$ Eddington (Joseph et al., 1976), $\delta$ two stream (Schaller, 1979) or exponential kernel (Wang, 1972) assume that the intensity diffracted forward is transmitted directly; they differ in the assumption of the fraction of intensity scattered into the foreward peak. In the double- $\delta$ Eddington approximation we proceed in two steps. The original phase function $\Gamma(\theta)$ is first truncated (Potter, 1970) by extrapolation from outside the peak (we chose $\theta=10^{\circ}$ ); the remainder of the phase function is left intact. The new phase function $\Gamma^{\prime}(\theta)$ is considerably smoother but still highly asymmetric. We thus follow the procedure that Wang (1972) applied to the exponential kernel: the part of the new phase function $\Gamma^{\prime}(\theta)$ which is assumed to be directly transmitted is chosen so that the integral of $\Gamma(\theta)$ from 90 to $180^{\circ}$ is conserved. Finally, we obtained a phase function such that the integrals

and

$$
\int_{0}^{10^{\circ}} \Gamma(\theta) d(\cos \theta) \quad \text { [forward scattering] }
$$

$$
\int_{90^{\circ}}^{180^{\circ}} \dot{\Gamma}(\theta) d(\cos \theta) \quad \text { [backward scattering] }
$$

are approximately conserved. The improvement is particularly important for strong absorption for which cases the $\delta$-Eddington approximation sometimes gives unrealistic results.

The combination of scattering and molecular absorption is handled by a technique based on a probability distribution of the amount of absorber. This technique enables us to calculate the mean amount of absorbing gas encountered by the radiation emerging at a given level since entering the atmosphere. The problem is then reduced to the standard problem of computing the attenuation of the solar beam in a clear atmosphere; absorption by water vapor, carbon dioxide, ozone, liquid water and aerosols is calculated in a simple way using accurate band models. The initial spectral data are extracted from the Lowtran 3b model (Selby et al., 1976). Using a single interval for the whole solar spectrum, Fouquart and Bonnel (1980) obtained the heating rate with an accuracy of the order of a few percent when compared to the calculations using high spectral resolution.

\section{d. Longwave radiation}

The method used to compute longwave radiation has been described by Morcrette (1978) where tests of accuracy can be found. Globally, the maximum discrepancy for the cooling rate of a clear atmosphere is less than $1 \mathrm{~K} \mathrm{day}{ }^{-1}$ when compared to Rodgers and Walshaw (1966). In cloudy conditions when the radiative divergence is considerably enhanced by the liquid water absorption, the percentage error is strongly decreased. Eq. (3.2) where the last term is replaced by $\left(1-\omega_{y}\right) B_{\nu}(\tau)$ is still solved by means of the Eddington approximation. To keep simple analytical solutions we had to assume that $B_{v}(\tau)$ is linear with respect to $\tau$ in each layer. Following Wiscombe (1976), the average error in $B_{\nu}(\tau)$ for layers of around $1 . \mathrm{km}$ thick is roughly less than $5 \%$. As the atmosphere is divided in 24 layers (six. in the cloud), the actual error must be considerably smaller.

The transmission functions for water vapor and carbon dioxide are expressed by a sum of exponentials, i.e.,

$$
T_{\Delta \nu}(u) \approx \sum_{i=1}^{5} a_{i} \exp \left(-k_{i} u\right)
$$

where $u$ is the amount of absorber.

The spectral data for the $6.3 \mu \mathrm{m}$ and rotation band of water vapor and the $\nu_{2}$ band of carbon dioxide near $15 \mu \mathrm{m}$ have been extracted from Rodgers and Walshaw (1966). The so-called $e$-type absorption which occurs in the atmospheric window is taken into account, using mean absorption coefficient computed from Roberts et al. (1976):

$$
T_{\Delta \nu}^{e}(u)=\exp \left(-C_{\Delta \nu} u e\right)
$$

where $e$ is the water vapor partial pressure. Altogether 25 spectral intervals are used for the longwave range. Because of the major importance of the window emission in a cloudy atmosphere, eight spectral intervals lie between 800 and $1200 \mathrm{~cm}^{-1}$.

In all spectral intervals, the accuracy of fit of Eq. (3.4) is better than $1 \%$. Since we are mostly interested in cloudy cases for which the liquid water absorption is large, a percentage error of $1 \%$ for the gaseous absorption is likely to be of little consequence to the overall radiation fields.

Water vapor and carbon dioxide absorption overlap in the range $560-760 \mathrm{~cm}^{-1}$. Since the water vapor and carbon dioxide spectra are uncorrelated, the multiplication property (Goody, 1964) can be applied:

$$
T_{\Delta \nu} \text { (overlap) } \approx T_{\Delta \nu}\left(\mathrm{H}_{2} \mathrm{O}\right) T_{\Delta \nu}\left(\mathrm{CO}_{2}\right) \text {. }
$$

For this particular interval, $T_{\Delta v}\left(\mathrm{CO}_{2}\right)$ is calculated from (3.4) while the water vapor transmission is 
represented by a single mean absorption coefficient. This, of course, is an approximation. Nevertheless, as noted by Goody (1964), it works quite well even for weak absorption and the corresponding error must be insignificant since the cloud is nearly black in this spectral region.

Ozone absorption is neglected in the longwave computation as it acts only in the higher atmosphere.

\section{An experimental reference case}

The first test of the diagnostic version of our model consists in comparing its results against those given by Lilly (1968), on the basis of the same experimental data. These data refer to persistent Californian stratocumulus with

$$
\left.\begin{array}{rl}
H & =620 \mathrm{~m}, \quad h=307.02 \mathrm{~kJ} \mathrm{~kg}^{-1}, \\
\Delta h & =5.7 \mathrm{~kJ} \mathrm{~kg}^{-1} \\
z_{C} & =345 \mathrm{~m}, \quad \sigma=7.8 \mathrm{~g} \mathrm{~kg}^{-1}, \Delta q=4.8 \mathrm{~g} \mathrm{~kg}^{-1}
\end{array}\right\}
$$

With his data, Lilly estimated the radiative heat loss $R L_{T}-R L_{H}=88 \mathrm{~W} \mathrm{~m} \mathrm{~m}^{2}$ and $R S_{T}-R S_{H}=-22$ $\mathrm{W} \mathrm{m}^{-2}$. In his original model, there is no radiative divergence in the mixed layer and $R L_{S}=R L_{H}$, $R S_{S}=R S_{H}$. To maintain a unique basis for comparison we adjusted the liquid water path (by means of the "diabatism coefficient" $A$ ) and the sun zenith

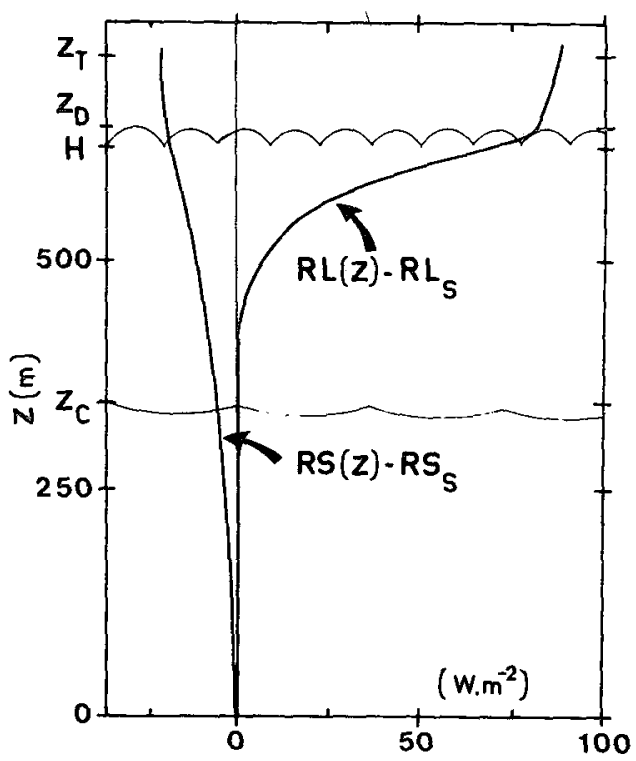

Fig. 2. Distributed shortwave $R S(z)-R S_{S}$ and longwave $R L(z)-R L_{S}$ flux profiles for Lilly's (1968) experimental reference case: $H=620 \mathrm{~m}, z_{C}=345 \mathrm{~m}, h=307.2 \mathrm{~kJ} \mathrm{~kg}^{-1}, \sigma=7.8$ $\mathrm{g} \mathrm{kg}^{-1}, \Delta h=5.7 \mathrm{~kJ} \mathrm{~kg}^{-1}$ and $\Delta q=4.8 \mathrm{~g} \mathrm{~kg}^{-1}$. These two profiles have been fitted to obtain the overall radiative differences $R S_{T}-R S_{S}$ and $R L_{T}-R L_{S}$ equal to -22 and $88 \mathrm{~W} \mathrm{~m}^{-2}$, respectively, as estimated by Lilly. Thus we take $W=13.5 \mathrm{~g} \mathrm{~m}^{-2}, \tilde{r}_{e}$ $=5.6 \mu \mathrm{m}, \tau_{N}=3.6$ and $\theta_{0}=40^{\circ}$. We assume $z_{T}-H=100 \mathrm{~m}$ and $z_{D}-H=20 \mathrm{~m}$.

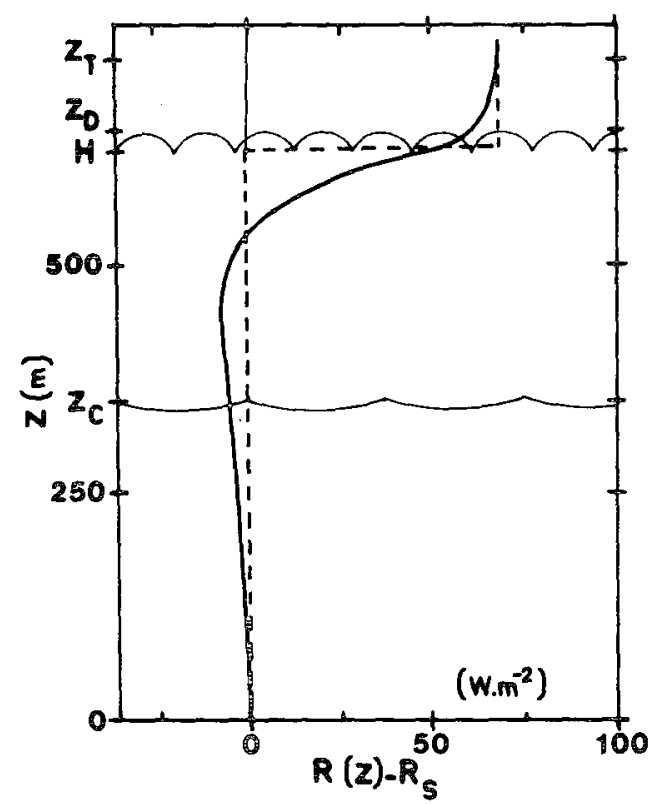

Frg. 3. Distributed total radiative flux profiles $R(z)-R_{S}$ deduced from Fig. 2 (solid line) and the Lilly discontinuous flux profile (dashed line) with the same radiative overall difference $R_{T}-R_{S}=66 \mathrm{~W} \mathrm{~m} \mathrm{~m}^{-2}$.

angle $\theta_{0}$, so that we get the same overall radiative differences for both $R L_{T}-R L_{H}$ (Lilly) $=R L_{T}-R L_{S}$ (present model) and $R S_{T}-R S_{H}$ (Lilly) $=R S_{T}-R S_{S}$ (present model). If $b=2 \mu \mathrm{m}$ in $r_{m}$, a fit is obtained with $A=0.17$ and $\theta_{0}=40^{\circ}$. Thus, the maximum liquid water ratio is $l_{H}=0.077 \mathrm{~g} \mathrm{~kg}^{-1}$ and the total liquid water path is $W=13.5 \mathrm{~g} \mathrm{~m}^{-2}$. The visible cloud optical thickness is $\tau_{N}=3.6$ for an average effective mean radius $\bar{r}_{e}=5.6 \mu \mathrm{m}\left(r_{e}=3.4 \mu \mathrm{m}\right.$ at cloud base and $6.8 \mu \mathrm{m}$ at cloud top). Furthermore, we set the thickness of the inversion layer $z_{T}-H$ $=100 \mathrm{~m}$ and the thickness of the cloud turrets $z_{D}-H=20 \mathrm{~m}$.

The corresponding solar and infrared flux profiles are shown in Fig. 2. Absorption of solar radiation causes warming everywhere in the mixed layer, whereas there is strong infrared cooling, mostly near the cloud top and within the inversion layer. The global net radiative flux $R(z)$ (solid line) is shown in Fig. 3, where we also have reproduced for comparison the radiative flux given in Lilly (dashed line). What comes out clearly is the nonlinear variation of $R(z)$ within the cloud, in contrast to the discontinuous profile in the other model.

As expected from (2.5) and (2.3), the effect of this nonlinearity can be seen in the profiles of the turbulent fluxes $F_{h}(z)$ and $F_{s v}(z)$ which are shown in Figs. 4 and 5 (solid line) and compute from Deardorff's (1976) surface flux values $\left[\left(F_{h}\right)_{S}=32.9\right.$ $\mathrm{W} \mathrm{m} \mathrm{m}^{-2}$ and $\left.L\left(F_{\sigma}\right)_{S}=28.3 \mathrm{~W} \mathrm{~m}^{-2}\right]$. In these figures, the dashed lines also refer to the discontinuous Lilly profile. In Fig. 5 the largest difference between 


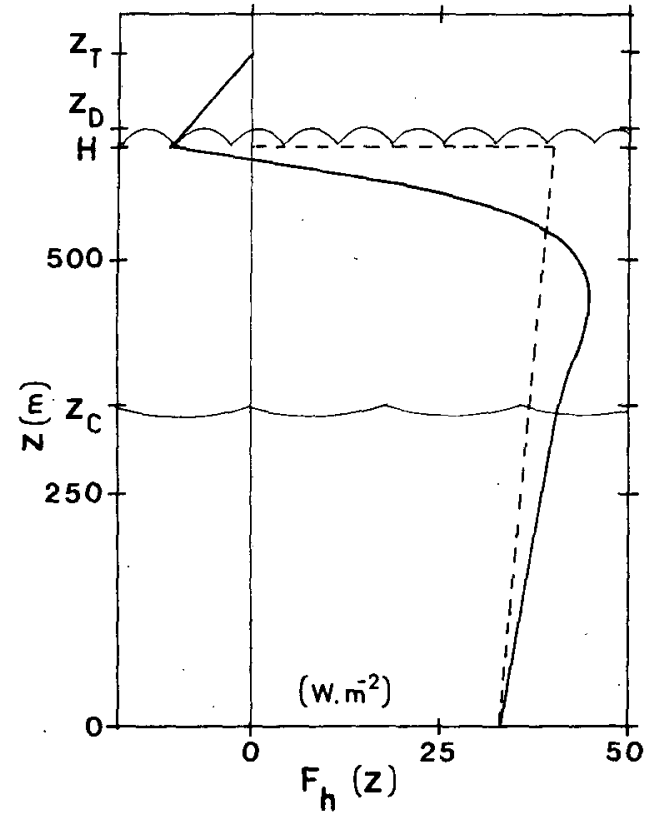

FIG. 4. From the experimental reference case (Fig. 2), comparison of the turbulent $F_{h}(z)$ flux profiles deduced from the algorithm of Section (2.4) with $k=0.2,\left(F_{h}\right)_{S}=32.9 \mathrm{~W} \mathrm{~m}^{-2}$ and $L\left(F_{\sigma}\right)_{S}=28.2 \mathrm{~W} \mathrm{~m}^{-2}$ : (a) for the distributed radiative flux profile (solid line), (b) for the schematic flux profile of Lilly (dashed line).

the two curves is seen near the cloud top where the turbulent flux of $F_{s z}(z)$ is negative in our model instead of being strongly positive as in Lilly. This is a very important point: as we can see in Fig. 5, our formulation preserves the negative buoyancy flux which is necessary to promote entrainment at cloud top (Deardorff and Businger, 1980). On the contrary, in Lilly (1968) no compensating zone of negative buoyancy flux appears below $H$ in Fig. 5. Thus, his entrainment process appears to lack a physical basis.

The intrusion of cloud turrets within the transition zone causes large fluctuations in the liquid water mixing ratio. Consequently, between $z_{D}$ and $H, F_{s v}$ must be larger than the values shown schematically in Fig. 5 (thick dashed line above $H$ ). However, the intensity of the corresponding jump is irrelevant in our model since, in (2.6) we only consider the budget of the transition layer as a whole.

For the same meteorological situation in which the data of Section 4 were collected, Lilly estimated (from soundings) the minimum and maximum entrainment rates $w_{e}$ to be $0.32 \mathrm{~cm} \mathrm{~s}^{-1}$ and $0.40 \mathrm{~cm} \mathrm{~s}^{-1}$. Our computational procedure applied to the nonlinear profile $R(z)$ gives $w_{e}=0.39 \mathrm{~cm} \mathrm{~s}^{-1}$, between the two preceding limits. Surprisingly, when applied to the radiative Lilly profile our computation procedure gives the same $w_{e}$ value $\left(0.39 \mathrm{~cm} \mathrm{~s}^{-1}\right)$. This coincidence calls for further study.

The radiative fluxes appear only in (2.11) in the calculation of $w_{e}$. With the particular data set of the present Section, it occurs that the sum of the two last terms in (2.11) is nearly zero (see Fig. 3). Moreover, since the overall difference $R_{T}-\boldsymbol{R}_{S}$ was fitted to Lilly's value $J_{R}, J$ and $w_{e}$ are nearly the same in the two cases. This equality occurs in spite of obvious differences in the turbulent fluxes $F_{h}(z)$ and $F_{s v}(z)$ and in spite of the displacement of the turbulent kinetic energy destruction zone from the base (Lilly, 1968) to the top (present model) of the cloud (dotted areas in Fig. 5).

The purely fortuitous equality observed between the two values of $w_{e}$ simply expresses an overall compensation between infrared cooling and solar warming in the cloud. Figs. 2 and 3 show that this compensation is only realized globally and not locally. The infrared cooling is concentrated near the top, whereas the solar warming is rather uniformly distributed within the cloud.

To emphasize further how accidental this compensation is, we calculated $w_{e}$ for no solar flux $\left[R S(z)=0\right.$ and $\left.R L_{T}-R L_{S}=88 \mathrm{~W} \mathrm{~m}^{-2}\right]$. In this case, according to our computational procedure, the $w_{e}$ values are quite different: $0.44 \mathrm{~cm} \mathrm{~s}^{-1}$ with our $R L(z)$ profile in Fig. 2 versus $0.51 \mathrm{~cm} \mathrm{~s}^{-1}$ for Lilly schematic profile.

In spite of the vertical discretization which is needed to calculate accurately the net radiative fluxes within the mixed layer, our model keeps the main features of Lilly's. It can thus be used to assess the cloud response to radiative forcing. The present result shows that there is strong coupling between radiative and turbulent fluxes. The accidental agreement between the two entrainment

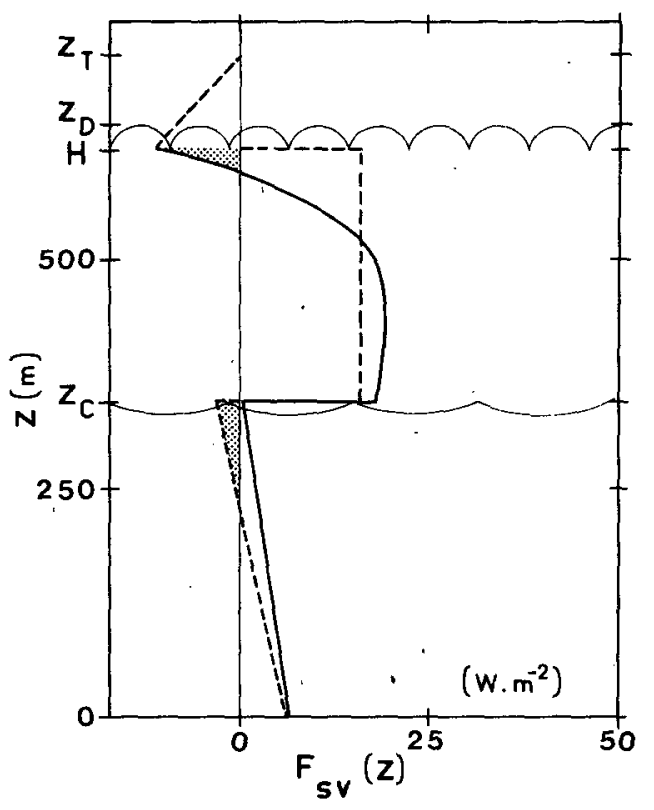

Fig. 5. Calculated turbulent $F_{s v}(z)$ flux profiles for the two cases defined in Fig. 4 
rates is evidence of the important role played by solar heating. This is a new result to be stressed.

\section{Sensitivity tests}

The radiative fluxes basically depend on the height of the cloud, the temperature and humidity proniles, the surface temperature and the cloud microphysics (liquid water content and drop size distribution).

In the present context we are mainly interested in the cloud microphysics, which affects the cloud dynamics only through the radiative fluxes. In this Section, we investigate the sensitivity of the entrainment rate and turbulent fluxes to the liquid water content and the drop size distribution. In order to maintain everything constant but the cloud microphysics, we find it convenient to simulate large variations in liquid water path by varying the coefficient $A$.

\section{a. Influence of the liquid water path}

Figs. $6 a$ and $6 \mathrm{~b}$ show the dependence of the radiative fluxes upon the liquid water path $W$ which varies from 4 to $64 \mathrm{~g} \mathrm{~m}^{-2}$ as the diabatism coefficient $A$ rises from 0.05 to 0.8 . At cloud top, the liquid water mixing ratio $l_{H}$ lies between 0.022 and $0.36 \mathrm{~g} \mathrm{~m}^{-2}$. These values are typical of stratocumulus (Zdunkowski and Crandall, 1971). For each value of $A$ we adjusted the parameter $b$ in $r_{m}$ so as to keep the average effective radius $\bar{r}_{e}$ equal to $5.6 \mu \mathrm{m}$ as in Section 4 . By doing so, we maintain the drop spectrum unchanged while varying $W$ by means of the param-

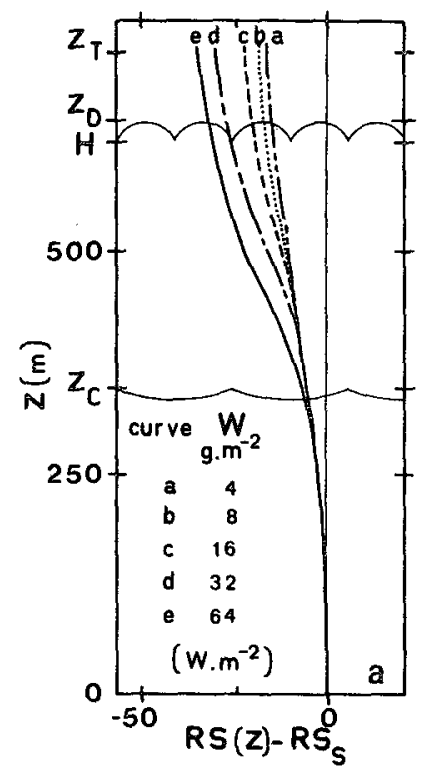

FIG. 6a. Shortwave flux $R S(z)-R S_{S}$ profiles calculated for the experimental reference case as a function of $W$ (from 4 to $64 \mathrm{~g} \mathrm{~m}^{-2}$ ) for $\bar{r}_{e}=5.6 \mu \mathrm{m}$ and $\theta_{0}=40^{\circ}$.

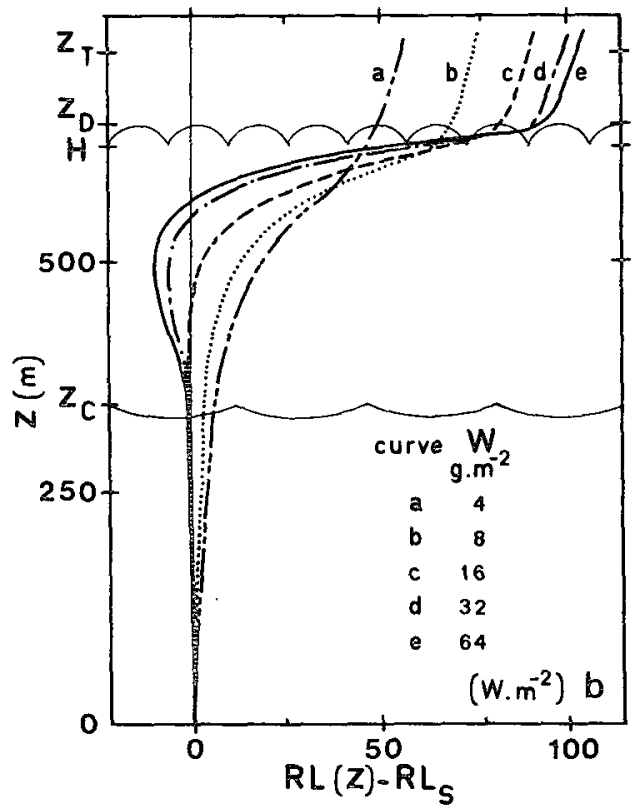

FIG. 6b. Longwave flux $R L(z)-R L_{S}$ profiles calculated for the experimental reference case as a function of $W$ (from 4 to $64 \mathrm{~g} \mathrm{~m}^{-2}$ ) for $\bar{r}_{e}=5.6 \mu \mathrm{m}$.

eter $A$. The visible optical thickness $\tau_{N}$ varies from $1.1(A=0.05)$ to $17(A=0.8)$.

The total shortwave radiative difference $R S_{T}$ $-R S_{S}$ (Fig. 6a) varies from -17 to $-37 \mathrm{~W} \mathrm{~m}^{-2}$ $\left(\theta_{0}=40^{\circ}\right)$, while the corresponding increase in the longwave radiative difference $R L_{T}-R L_{S}$ (Fig. 6b) is noticeably greater (from 59 to $102 \mathrm{~W} \mathrm{~m}^{-2}$ ). Thus, the global solar heating still corresponds to $\sim 30 \%$ of the infrared cooling. The cloud as a whole is therefore characterized by radiative cooling.

However, our model brings new results due to the consideration of the vertical distribution of the radiative fluxes within the cloud layer, a point of main concern. For weak $W$, the infrared cooling is weak and is distributed throughout the whole cloud. With increasing liquid water path, the infrared cooling grows and tends to be more and more localized near the cloud top, so that with large $W$ the limiting profile of the longwave radiation is roughly similar to the somewhat idealized profile of Lilly's model. On the contrary, the shortwave heating is always more or less uniformly distributed throughout the cloud. Thus, in the upper part of the cloud, solar heating partly compensates for the radiative cooling while near the cloud base it notably reinforces the infrared warming which appears when $W$ is large enough. This sequence of radiative profiles is clearly depicted in Figs. $6 \mathrm{a}$ and $6 \mathrm{~b}$ for different values of $W$ (curves a to e). As a consequence, the lower part of the cloud can be a local source of turbulent kinetic energy. This appears in Figs. 7 and 8 , where the $F_{h}(z)$ and $F_{s v}(z)$ profiles 


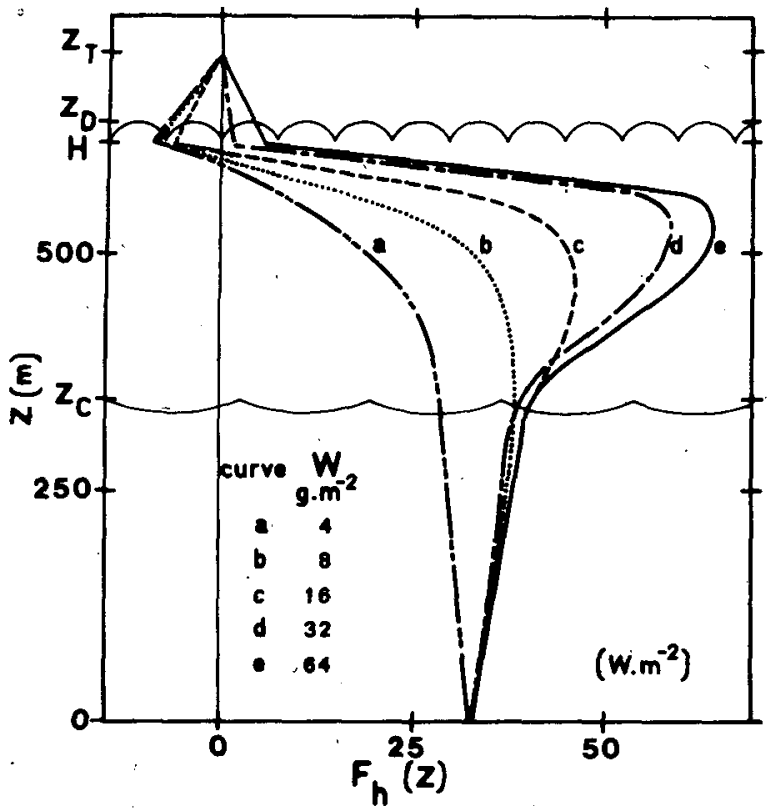

Fig. 7. Calculated turbulent flux $F_{h}(z)$ profiles corresponding to the radiative flux profiles depicted in Figs. $6 a$ and $6 \mathrm{~b}$.

are depicted for $\theta_{0}=40^{\circ}$ and where we note an increase of these two fluxes with $z$, above the cloud base, for $W>16 \mathrm{~g} \mathrm{~m}^{-2}$.

Because of the great sensitivity of the longwave fluxes to the liquid water path (Fig. 6b), the turbulent fluxes show large variability for small $W$ (Figs. 7 and 8). In these figures, the limiting profiles for large

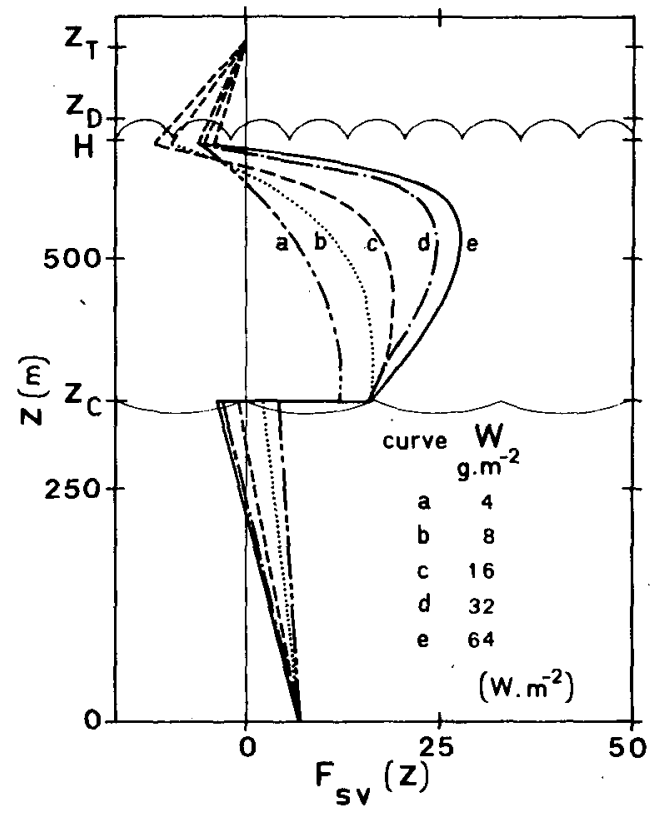

Fig. 8. Calculated turbulent flux $F_{s i}(z)$ profiles corresponding to the radiative flux profiles depicted in Figs. $6 \mathrm{a}$ and $6 \mathrm{~b}$.

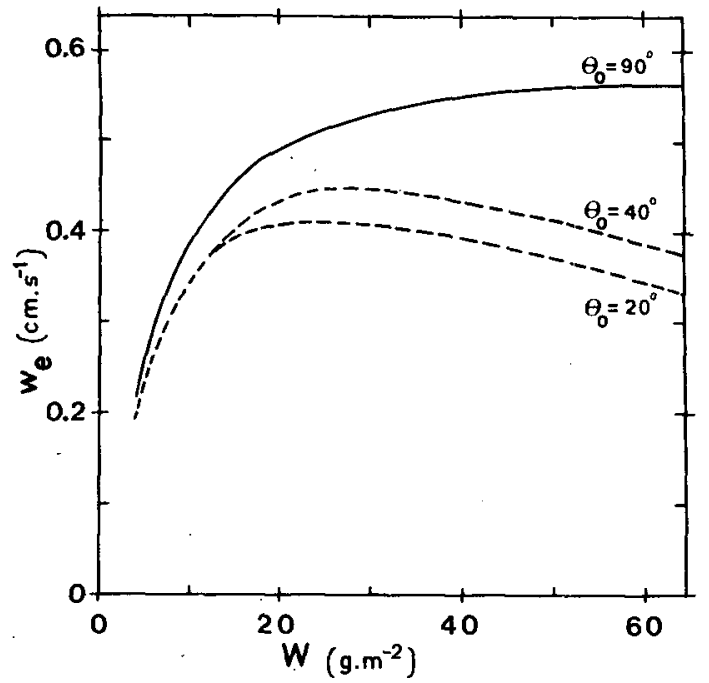

Fig. 9. Variation of the entrainment rate $w_{e}$ with $W$ for no solar flux $\left(\theta_{0}=90^{\circ}\right)$ and daytime $\left(\theta_{0}=40\right.$ and $\left.20^{\circ}\right)$ conditions for a constant drop spectrum $\left(\bar{r}_{e}=5.6 \mu \mathrm{m}\right)$.

$W\left(>64 \mathrm{~g} \mathrm{~m}^{-2}\right)$ tend towards the schematic linear profiles in Lilly's (1968) model.

With regard to the entrainment rate, $w_{e}$ is seen to increase rapidly with $W$ (Fig. 9) as long as $W$ remains low; this must be related to the longwave flux profiles in Fig. 6b. At low $W$ the solar heating is small at all values of $\theta_{0}$ and plays only a minor part in fixing $w_{e}$. The behavior of the infrared flux profiles at large $W$ leads to a saturation effect on $w_{e}$, which is nearly constant for zero solar flux $\left(\theta_{0}=90^{\circ}\right)$ and for $W>40 \mathrm{~g} \mathrm{~m}^{-2}$. This corresponds to a constant radiative term $J_{R}$ in (2.11).

Taking solar radiation into account reduces considerably the entrainment rate (via $J_{R}$ ) with large liquid water paths. For example, in Fig. 9, for $W$ $=64 \mathrm{~g} \mathrm{~m}^{-2}, w_{e}$ is reduced by $30 \%$ at $\theta_{0}=40^{\circ}$ and by $40 \%$ at $\theta_{0}=20^{\circ}$.

This sensitivity of the entrainment rate to the solar heating suggests a possible significant diurnal cycle in thick stratocumulus clouds over the sea. Our results also suggest an explanation of Paltridge's observation (1974b) of a fast dissipating stratocumulus cloud deck in the shadow of an upper altocumulus patch. Presumably, because of the weak inversion strength $\left(\Delta T=5 \mathrm{~K}\right.$ and $\left.\Delta q=5 \mathrm{~g} \mathrm{~kg}^{-1}\right)$, the cloud deck was nearly unstable with reference to the buoyancy flux instability criterion of Randall (1980b) and Deardorff (1980a). This is confirmed by the dissipation of the rest of the cloud half an hour later. Then the only problem is to explain why a reduction in shortwave flux should lead to a premature breakup. As seen in Fig. 9, for larger liquid water contents, the entrainment rate should be notably enhanced when the solar heating is suddenly reduced. Consequently, more parcels of dry and 
warm air are brought down into the cloud while the cooling is enhanced by the increase of radiative divergence, and by the evaporation of liquid water in those parcels which sink toward the cloud base as they become negatively buoyant with respect to their cloudy environment. This results in the rapid dissipation of the cloud.

In view of the above results, the comparison between our scheme and the others needs to be carried further since it appears that, neglecting solar heating, these schemes represent a limiting case for large $W$. In Fig. 10, we display as a function of $W$, the proportional difference $\Delta w_{e} / w_{e}=\left[w_{e}\right.$ (Lilly) $\left.-w_{e}\right] / w_{e}$, where $w_{e}$ is our value. The other value of $w_{e}$ is calculated using the schematic representation of the radiative flux profiles with the same total radiative difference $R_{T}-R_{S}$.

In the absence of solar flux $\left(\theta_{0}=90^{\circ}\right)$, with small $W$ (Fig. 10), Lilly's model greatly overestimate $w_{e}$ (by more than $50 \%$ for $W=4 \mathrm{~g} \mathrm{~m}^{-2}$ ), since the radiative divergence is distributed throughout the cloud layer (Fig. 6b) and since the contribution of the last integral in (2.11) is significant in our formulation. As $W$ increases, the discrepancy is reduced and becomes less than $5 \%$ when $W>30 \mathrm{~g} \mathrm{~m}^{-2}$.

The difference between the two schemes is notably reduced at low values of $\theta_{0}$ and small $W$. For larger $W$, the location of the longwave cooling is rather similar in the two models and the longwave flux contribution to the last term in (2.11) remains small. The difference between the two models appears only in the positive solar flux contribution to the last term in (2.11), making $w_{e}$ greater in our model $\left(\Delta w_{e}<0\right)$.

Finally, the agreement between the two schemes is good as long as the solar flux can be neglected and as long as the liquid water path is large enough to ensure intense infrared cooling at cloud top. Note that the coincidence obtained in Section 4 for the experimental reference case $\left(\Delta w_{e} \approx 0\right)$ corresponds to the point marked $\mathrm{C}$ in Fig. $10\left(W=13.5 \mathrm{~g} \mathrm{~m}^{-2}\right.$, $\left.\theta_{0}=40^{\circ}\right)$.

\section{b. Influence of the drop size distribution}

To simulate a change in the drop spectrum, all other factors being kept constant, we change the equivalent mean radius $\bar{r}_{e}$. The radiative and turbulent fluxes for $W=16 \mathrm{~g} \mathrm{~m}^{-2}\left(A=0.2\right.$ and $l_{H}$ $\left.=0.09 \mathrm{~g} \mathrm{~kg}^{-1}\right)$ are reproduced in Figs. $11 \mathrm{a}$ and $11 \mathrm{~b}$ for two extreme cases: case 1 with $\bar{r}_{e}=14 \mu \mathrm{m}$ (wide spectrum with $b=7 \mu \mathrm{m}$ ) and case 2 with $\bar{r}_{e}=3$ $\mu \mathrm{m}$ (narrow spectrum with $b=0.3 \mu \mathrm{m}$ ).

In the solar range, where the Mie parameter $x \gg 1$, the extinction efficiency is roughly constant $\left(Q_{\text {ext }} \approx 2\right)$ and the optical thickness is proportional to $\bar{r}_{e}^{-1}$. Consequently, the shortwave radiative divergence increases when $\bar{r}_{e}$ decreases (Fig. 11a). The behavior of the longwave radiative fluxes is a

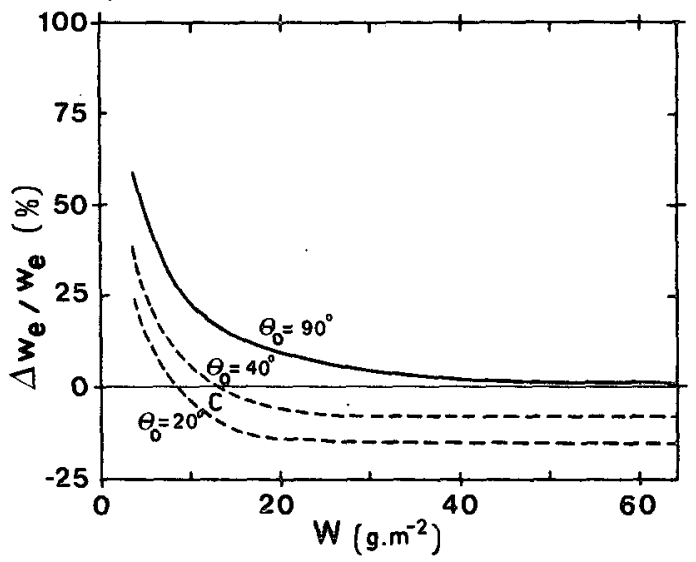

Fig. 10. Variation of the relative difference $\Delta w_{e} / w_{e}=\left[w_{e}\right.$ (Lilly) $\left.-w_{e}\right] / w_{e}$ with $W$, for a constant drop spectrum $\left(\bar{r}_{e}=5.6\right.$ $\mu \mathrm{m}$ ), where $w_{e}$ results from the present model (Fig. 9) and the other value of $w_{e}$ is calculated using the same radiative difference $R_{T}-R_{S}$ as in our model but using the Lilly's schematic representation of the radiative divergence.

little more surprising if we consider the commonly accepted statement that emissivity is nearly independent of the drop spectrum (Paltridge, 1974a; Stephens, 1978b). However, this statement is based on the assumption of small or even moderate $x$, in which the absorption efficiency is approximately linear with respect to $x$ and the mass absorption coefficient is roughly independent of $r$. If scattering is neglected the radiative cooling is a function of the absorption coefficient $\left(Q_{\text {abs }}\right)$ only and it is thus nearly independent of the drop spectrum for small or intermediate particles. For larger particles, on the contrary, $Q_{\text {abs }}$ tends to unity and the mass absorption is proportional to $\bar{r}_{e}^{-1}$. Then, the radiative cooling increases when decreasing $\bar{r}_{e}$ as it is the case for the solar range. Following Morcrette (1978), the cooling becomes independent of the size for $r \leqslant 6$ $\mu \mathrm{m}$. However, in the present case, the effective mean radius $r_{e}$ increases from bottom to top so that the longwave flux remains dependent on the equivalent mean radius $\bar{r}_{e}$ for particles $<6 \mu \mathrm{m}$.

Fig. 12 shows the variation of $w_{e}$ as a function of $\bar{r}_{e}$ for various values of $W$ and for $\theta_{0}=90^{\circ}$ (the limits of the visible optical thickness $\tau_{N}$ are also shown on each curve in Fig. 12). As expected, the entrainment rate is independent of $\bar{r}_{e}$ for large $W$, in which cases the cloud is black whatever $\bar{r}_{e}$ is. That is consistent with the statement of increasing opacity with decreasing $\bar{r}_{e}$. For the thinnest clouds $w_{e}$ increases when $\bar{r}_{e}$ decreases and it may be seen that the most pronounced variation of $w_{e}$ is $\sim 25 \%$ with $W=8 \mathrm{~g} \mathrm{~m}^{-2}$ when $\bar{r}_{e}$ varies from 5 to $10 \mu \mathrm{m}$.

The behavior of $w_{e}$ as a function of $\tilde{r}_{e}$ is modified when one takes into account the absorption of solar radiation (Fig. 13). Since liquid water absorbs much more in the infrared than in the visible, the longwave 


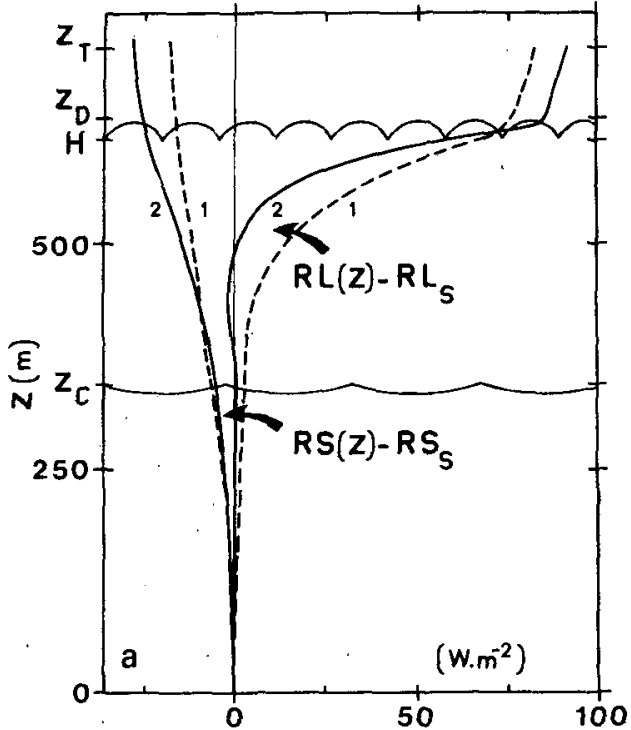

FIG. 11a. Comparison of the shortwave $R S(z)-R S_{S}\left(\theta_{0}=40^{\circ}\right)$ and longwave $R L(z)-R L_{S}$ flux profiles for a constant liquid water path ( $\left.W=16 \mathrm{~g} \mathrm{~m}^{-2}\right)$ and two drop spectra: case 1 with $\bar{r}_{e}=14 \mu \mathrm{m}(b=7 \mu \mathrm{m})$, case 2 with $\bar{r}_{e}=3 \mu \mathrm{m}(b=0.3 \mu \mathrm{m})$.

radiative heat loss is saturated whereas the shortwave absorption continues to increase. This is the reason why the slopes of the curves $w_{e}=f\left(\bar{r}_{e}\right)$ reverse at $W \geqslant 32 \mathrm{~g} \mathrm{~m}^{-2}$, in contrast to Fig. 12 .

The results of our model reported in this Section illustrate its sensitivity to the liquid water path $W$ as the main factor. The drop size distribution is also a significant parameter, though much less efficient than $W$. However, in real clouds, $W$ and the drop spectrum do not vary independently. Experimental

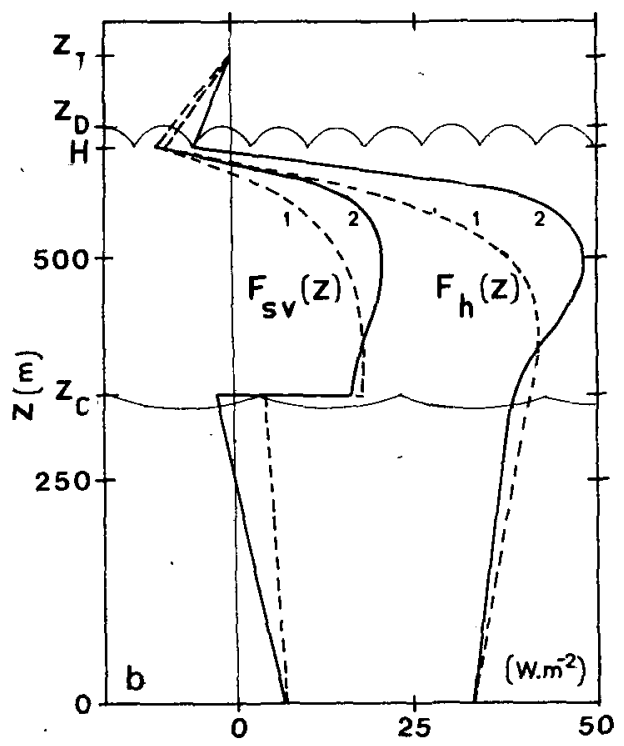

Fig. 11b. Comparison of the turbulent flux $F_{h}(z)$ and $F_{s c}(z)$ profiles for the two cases defined in Fig. 11a.

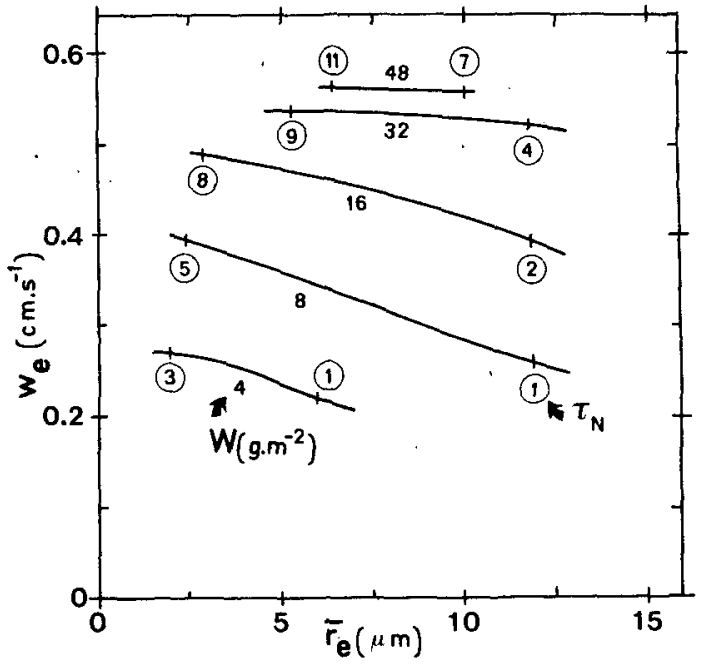

FlG. 12. Variation of the entrainment rate $w_{e}$ as a function of $\bar{r}_{e}$ and of $W$ (from 4 to $48 \mathrm{~g} \mathrm{~m}^{-2}$ ) for zero solar flux $\left(\theta_{0}=90^{\circ}\right.$ ). The extreme values of the visible optical thickness are circled.

data (Paltridge, 1974a; Stephens et al., 1978) show that high liquid water contents are generally associated with large drops, so it is likely that the sensitivity of $w_{e}$ to solar heating for simultaneous changes in both $W$ and $\bar{r}_{e}$ will be less than expected from Fig. 9. Indeed, for large $W$, since $\bar{r}_{e}$ increases with $W$, the optical thickness $\tau_{N}$ increases more slowly than assumed in Fig. 9. Moreover the foreward peak of the scattering phase function increases with $\dot{r}_{e}$, which further decreases the absorption of solar radiation. This result in smaller slopes of the curves $w_{e}=f(W)$ for large $W$ in Fig. $9\left(\theta_{0}=40\right.$ and $\left.20^{\circ}\right)$. However, the solar heating influence on the cloud deck remains qualitatively the same.

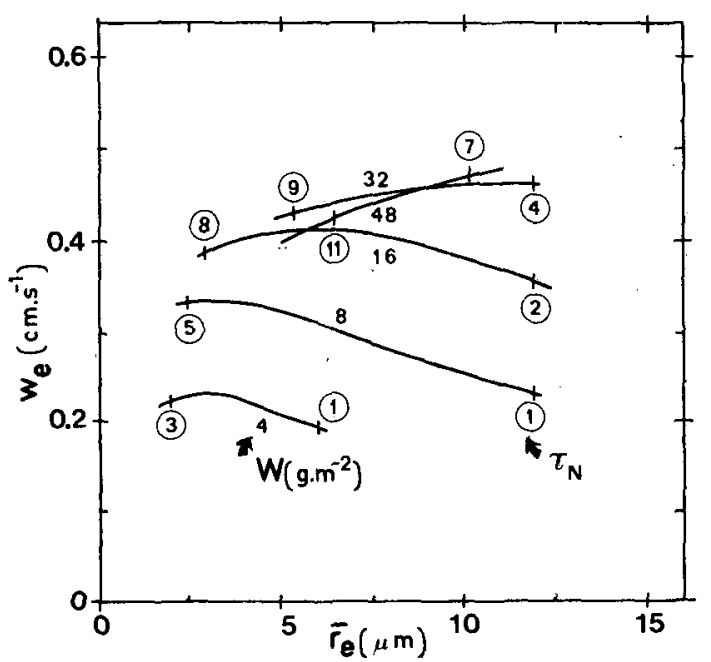

FIG. 13. Variation of the entrainment rate $w_{e}$ as a function of $\bar{r}_{e}$ and of $W$ (from 4 to $48 \mathrm{~g} \mathrm{~m}^{-2}$ ) for daytime conditions $\left(\theta_{0}\right.$ $=40^{\circ}$ ). The extreme values of the visible optical thickness are circled. 
Radiative cooling and heating are strongly dependent on the liquid water path; hence for constant $W$, the impact of a change in the distribution of $l(z)$ can be estimated from the liquid water content integrated between $z_{C}$ and $H$. For example, if we keep the $l(z)$ profile as in Fig. 1, the lowering of the level of maximum liquid water mixing ratio causes an increase of the integrated liquid water path in the part of the cloud between $z_{C}$ and $H$ at the expense of its upper part (between $H$ and $z_{D}$ ). Consequently, the total radiative divergence $R_{T}-R_{S}$ is unchanged while the last integral in (2.11) increases, causing a decrease in the production of turbulent kinetic energy within the cloud and reducing $w_{e}$.

\section{Conclusion}

In this paper, we report the results of complete radiative transfer calculations as a function of cloud characteristics in order to investigate the effect of the radiative fluxes on turbulent fluxes and entrainment velocity. We find that in clouds, strong divergence of the radiative fluxes results in a nonlinear structure of the turbulent $F_{h}(z)$ and $F_{s v}(z)$ flux profiles. This structure allows a negative buoyancy flux to exist at the inversion level, thus correcting misleading features of Lilly-type models.

The sensitivity tests stress the central role played by the integrated liquid water content and the secondary but non-negligible role of the drop size distribution.

Our result confirm that Lilly's (1968) original representation of the radiative divergence corresponds roughly to the case of thick clouds with large liquid water paths, neglecting the shortwave fluxes. Moreover, in such clouds the radiative cooling is localized near the cloud top and its saturation implies that the turbulent $F_{h}(z)$ and $F_{s v}(z)$ flux profiles are also saturated. Consequently, the entrainment rate remains nearly constant when the liquid water path varies.

A particularly interesting result is the sensitivity of the model to the shortwave heating which notably reduces the entrainment rate in thick clouds. As noted by a referee, it is worthwhile to point out that measurements of solar absorptance hint at even larger values than those calculated in the present paper. This suggests a significant diurnal cycle in thick stratocumulus clouds. However, shortwave absorption acts not only to reduce the total radiative loss of the cloud layer. Since it is not only localized at cloud top, but distributed throughout the cloud, it participates as a source of turbulent kinetic energy in the lower part of the cloud, while acting as a sink near the cloud top.

In thin clouds, shortwave absorption is weak, so that it plays a negligible part in the turbulence. As for longwave cooling, it is distributed throughout the cloud layer instead of being simply localized at cloud top as in thick clouds; furthermore, it depends strongly on the microphysical cloud properties. As a consequence, the turbulent fluxes $F_{h}(z)$ and $F_{s v}(z)$ are highly sensitive to liquid water path and the entrainment rate rapidly increases with this parameter.

Cloud response to radiative forcing clearly shows that the radiative fluxes should be calculated as functions of the integrated liquid water content instead of being prescribed in advance. This parameter should therefore be accurately determined experimentally. However, in our future work the exhaustive flux computations will need to be simplified. A simple way would consist of a direct parameterization of the cloud's infrared emissivity as a function of the liquid water path: $\epsilon_{\mathrm{L}}=1-\exp (-a W)$ (Paltridge, 1974b; Stephens, 1978b; Morcrette, 1978).

Similar parameterizations of shortwave absorption should be possible, but a satisfactory one has not yet been found.

Acknowledgments. The authors wish to thank Drs. G. L. Stephens and W. H. Schubert for helpful comments on an earlier version of this paper. Acknowledgments are also due to Profs. J. Lenoble and R. G. Soulage for their continued assistance and advice and to Prof. Morel for initiating this work. This research was supported by the Centre National de la Recherche Scientifique under Contracts RCP 413 and ATP (PIRDES) 3986.

\section{REFERENCES}

Ball, F. K., 1960: Control of inversion height by surface heating. Quart. J. Roy. Meteor. Soc., 86, 483-494.

Deardorff, J. W., 1976: On the entrainment rate of a stratocumulus-topped mixed layer. Quart. J. Roy. Meteor. Soc., 102, 563-582.

, 1980a: Cloud top entrainment instability. J. Atmos. Sci., $37,131-147$.

-, 1980b: Stratocumulus-topped mixed layer derived from a three dimensional model. Bound-Layer Meteor., 18, $495-527$.

_- and J. A. Businger, 1980: Comments on "Marine stratocumulus convection. Part I: Governing equations and horizontally homogeneous solutions." J. Atmos. Sci., 37, 481-482.

Feigelson, E. M., 1964: Light and Heat Radiation in Stratus Clouds. English version by Israel Program for Scientific Translations, Jerusalem, 1966, $245 \mathrm{pp}$.

Fouquart, Y., and B. Bonnel, 1980: Computations of solar heating of the earth's atmosphere: A new parameterization. Contrib. Atmos. Phys., 53, 35-62.

Goody, R. M., 1964: Atmospheric Radiation, 1. Theoretical Basis. Clarendon Press, 436 pp.

Joseph, J. H., W. J. Wiscombe and J. Weinman, 1976: Solar flux transfer through turbid atmospheres evaluated by the $\delta$ Eddington approximation. J. Atmos. Sci., 33, 2452-2459.

Kahn, P. H., and J. A. Businger, 1979: The effect of radiative flux divergence on entrainment of a saturated convective boundary. Quart. J. Roy. Meteor. Soc., 105, 303-306.

Kraus, H., and E. Schaller, 1978a: Steady-state characteristics of inversions capping a well-mixed planetary boundary-layer. Bound.-Layer Meteor., 14, 83-104. 
$\longrightarrow$, and $-1978 \mathrm{~b}$ : A note on the closure in Lilly-type inversion models. Tellus, 30, 284-288.

Lenoble, J., 1977: Standard procedures to compute atmospheric radiative transfers in scattering atmospheres. Proc. IAMAP Radiative Commission, NCAR, Boulder, 125 pp. (see pp. 93-96).

Lilly, D. K., 1968: Models of cloud-topped mixed layers under a strong inversion. Quart. J. Roy. Meteor. Soc., 94, 292-309.

Morcrette, J. J., 1978: Infrared fluxes in stratiform model clouds. Contrib. Atmos. Phys., 51, 338-351.

Paltridge, G. W., 1971: Solar and thermal radiation flux measurements over the east coast of Australia. J. Geophys. Res. , 76, 2857-2865.

—_, 1974a: Infrared emissivity short-wave albedo and the microphysics of stratiform water clouds. J. Geophys. Res., 79, 4053-4058.

— 1 1974b: Atmospheric radiation and the gross character of stratiform cloud. J. Atmos. Sci., 31, 244-250.

Platt, C. M. R., 1976: Infrared adsorption and liquid water content in stratocumulus cloud. Quart. J. Roy. Meteor. Soc., $102,533-561$.

Potter, J. E., 1970: The delta-function approximation in radiative transfer theory. J. Atmos. Sci., 27, 943-949.

Randall, D. A., 1980a: Entrainment into a stratocumulus layer with distributed radiative cooling. J. Atmos. Sci., 37, $125-130$.

$\longrightarrow$, 1980b: Conditional instability of the first kind upside down. J. Atmos. Sci., 37, 125-130.

Roberts, R. E., J. E. A. Selby and L. M. Biberman, 1976: Infrared continuum absorption by atmospheric water vapor in the 8-12 $\mu \mathrm{m}$ window. Appl. Opt., 15, 2085-2090.

Rodgers, C. D., and C. D. Walshaw, 1966: The computation of infrared cooling rate in planetary atmospheres. Quart. J. Roy. Meteor. Soc., 92, 67-92.

Schaller, E., 1979: The $\delta$-two stream approximation in radiative flux calculations. Contrib. Atmos. Phys., 52, 17-26.

Schubert, W. H., 1976: Experiments with Lilly's cloud toppedmixed layer model. J. Atmos. Sci., 33, 436-446.

__ J. S. Wakefied, E. J. Steiner and S. K. Cox, 1979: Marine stratocumulus convection. Part I: Governing equations and horizontally homogeneous solutions. J. Atmos. Sci., 36, 1286-1307.

Selby, J. E. A., E. P. Shettle and R. A. Mc. Clatchey, 1976: Atmospheric transmittance from 0.25 to $28.5 \mu \mathrm{m}$. Suppl. Lowtran 3B, AFGL-TR 76 0258, Environ. Res. Pap., No. $587,77 \mathrm{pp}$.

Shettle, E. P., and J. A. Weinman, 1970: The transfer solar irradiance through inhomogeneous turbid atmospheres evaluated by Eddington's approximation. $J$. Atmos. Sci., 27, 1048-1055.

Stephens, G. L., 1978a: Radiation profiles in extended water clouds. I: Theory. J. Atmos. Sci., 35, 2111-2122.

__ 1978b; Radiation profiles in extended water clouds. II: Parameterization schemes. J. Atmos. Sci., 35, 2123-2132.

__ G. W. Paltridge and C. M. R. Platt, 1978: Radiation profiles in extended water clouds, III: Observations. $J$. Atmos. Sci., 35, 2133-2141.

Wang, L., 1972: Anisotropic non conservative scattering in a semi-infinite medium. Astrophys. $J$., 174, 671-678.

Wiscombe, W. J., 1976: Extension of the doubling method to inhomogeneous sources. J. Quant. Spectros. Radiat. Transfer, 16, 477-489.

- , and J. H. Joseph, 1977: The range of validity of the Eddington approximation. Icarus, 32, 362-377.

Zdunkowski, G. W., and W. K. Crandall, 1971: Radiative transfer of infrared radiation in model clouds. Tellus, 23, 517-527. 